\title{
ene
}

\section{Epistemología de LA Profesión ENFERMERA}

\section{EPISTEMOLOGY OF THE NURSING PROFESSION}

\author{
Pavel Rodríguez-Bustamante1, Francisco Javier Báez-Hernández² \\ ${ }^{1}$ Hospital de Ginecopediatría, Instituto Mexicano del Seguro Social. Sonora, México. Maestro en \\ Enfermería. \\ 2 Facultad de Enfermería, Benemérita Universidad Autónoma de Puebla. Puebla, México.
} México. Doctor en Ciencias de Enfermería. 


\section{Resumen}

El marco epistemológico de la disciplina de enfermería, es resumido en dos estructuras de conocimiento (teórico y práctico), el primero enfocado a la ciencia y los elementos teóricos; el segundo considerado como el arte de la disciplina a través de las dimensiones prácticas y los patrones del conocimiento enfermero. El objetivo del presente artículo es describir y analizar el marco epistemológico de enfermería, para ello se contrastaron las propuestas estructurales de Fawcett, Smith y Liehr, y Kérouac en representación del conocimiento teórico, asimismo, se vincularon las dimensiones prácticas de enfermería con los patrones del conocimiento, para analizar el conocimiento práctico.

Palabras clave: conocimiento, enfermería, filosofía en enfermería, modelos de enfermería, teoría de enfermería.

\section{Abstract}

The epistemological framework of the nursing discipline is summarized in two knowledge structures (theoretical and practical). The first one focused on science and theoretical elements; the second one considered as the art of the discipline through the practical dimensions and the patterns of the nurse knowledge. The objective of this article is to describe and analyze the nursing epistemological framework, for this the structural proposals of Fawcett, Smith and Liehr, and Kérouac were contrasted in representation of the theoretical knowledge, likewise, the practical dimensions of nursing were linked with the knowledge patterns, to analyze the practical knowledge.

Key words: knowledge, nursing, philosophy nursing, models nursing, nursing theory. 


\section{INTRODUCCIÓN}

El debate acerca de la identidad de enfermería como ciencia y disciplina ha sido argumentado con vehemencia y fervor durante los últimos tiempos, en los que el principal tema en disputa es responder si la profesión es considerada o no como disciplina científica (1) (2). En sus inicios, enfermería emergió como ocupación, de la misma manera que la mayoría de las profesiones, por lo que se sostiene que "todas las profesiones son ocupaciones, pero no todas las ocupaciones son profesiones". En términos generales, éstas deben cumplir con las siguientes características esenciales: enseñanza formativa larga y rigurosa, contar con un paradigma relacionado a un cuerpo propio de conocimientos, reconocimiento legal con validez oficial, actuar a través de un código de ética, ejercicio autónomo de competencias específicas y por último, los profesionistas deben mostrar una conducta dirigida a satisfacer las necesidades de la sociedad (3).

\section{DesarRollo}

Es aprobado que el cuerpo de conocimientos de la disciplina se ha desarrollado y madurado crecientemente, quedando de manifiesto su naturaleza profesional (2). El presente artículo tiene como propósito, describir y analizar el marco epistemológico de enfermería, considerado el punto de partida para la génesis de la ciencia, el progreso y la clasificación del conocimiento (4). Se retomarán las dos dimensiones de enfermería propuestas por Kim, como directrices teóricas para el desarrollo argumentativo del escrito: 1) la ciencia de enfermería como base de conocimientos, constituida por los elementos teóricos relacionados a los fenómenos de interés disciplinario, integrada por la estructura epistémica del conocimiento y 2) el arte de enfermería, como base para la praxis y la aplicación creativa del conocimiento, integrada por las dimensiones de la práctica de enfermería y los patrones del conocimiento (5) (6).

\section{La ciencia de enfermería}

La ciencia del cuidado es concebida como un cuerpo creciente de ideas que puede caracterizarse por un conocimiento racional, sistemático, exacto, fiable y verificable (7), avalado por la investigación científica y el análisis lógico, cuyo objetivo radica en la creación de modelos y teorías que proporcionan marcos de referencia para perfeccionar la práctica diaria, a través de la descripción, predicción y el control de los fenómenos de interés disciplinarios (8).

Cada disciplina tiene un enfoque único y organizado para el desarrollo del 
conocimiento que dirige la investigación y la distingue de otros campos de estudio, comprender dicha organización o estructura disciplinaria, es un componente crucial para ampliar el aprendizaje y seguir el desarrollo cognitivo de las perspectivas teóricas de la profesión (6). En este sentido, el conocimiento teórico y sus funciones intelectuales se integran por los principios y elementos que impulsan el pensamiento y la comprensión de enfermería (9)(10), de modo que diversas autoras han creado propuestas estructurales que integran los componentes disciplinares indispensables para el estudio y progreso de la profesión (11), mismos que resumen y reúnen el marco epistemológico de enfermería, al lograr derivar elementos abstractos hasta lograr llegar a elementos concretos y precisos que validan las afirmaciones del conocimiento disciplinario (12)(13).

En lo sucesivo, para el desarrollo de este segmento del artículo se tomó como elemento rector la estructura jerárquica del conocimiento contemporáneo de Fawcett integrada por cinco niveles que parten de lo abstracto a lo concreto (14), en contraste con la estructura de la disciplina de enfermería de Smith y Liehr conformada por cinco niveles basados en el nivel de profundidad y de abstracción (6), al igual que los niveles de conceptualización de enfermería propuestos por Kérouac, quien clasifica el conocimiento en seis escuelas ubicadas dentro de los diversos paradigmas de enfermería (15), en consideración de que estas estructuras como marcos disciplinares organizan, clasifican y sintetizan el conjunto de saberes sustantivos a la disciplina.

Metaparadigma. Fawcett, Kérouac, y Smith y Liehr sostienen que éste es el componente de mayor abstracción y la estructura de base a partir de la cual el conocimiento puede evolucionar, puesto que incluye términos extremadamente generales y no direccionan actividades como el quehacer práctico e investigativo (14)(15)(6). Son Smith y Liehr quienes adicionan a este apartado el enfoque de la disciplina, como el estudio de las experiencias de salud humana y la curación a través del cuidado, ante la premisa de que la curación implica un proceso de cambio y evolución, en donde el cuidado es el camino a la curación (6). Así pues, desde esta óptica, la tetralogía metaparadigmática ancla y articula el sistema de engranes que en convergencia movilizan y permiten dirigir el actuar profesional mediante la identificación de los fenómenos únicos y específicos del cuidado.

Filosofías. De acuerdo a Fawcett, la función de este apartado radica en comunicar a los integrantes de la disciplina y a la sociedad, acerca de las creencias 
y valores que guían el actuar profesional

(14) a través de declaraciones alusivas a las diversas corrientes del pensamiento o paradigmas y maneras de ver los fenómenos disciplinarios (15). Si bien existen múltiples propuestas paradigmáticas, las tratadas en este artículo posibilitaron el contraste de ideas y su equiparación en función de las coincidencias identificadas en la direccionalidad, la manera de abordar y comprender a la persona y al mundo desde una perspectiva fragmentarista, hasta evolucionar aproximándose a una panorámica dirigida a la suma de partes y a la totalidad del individuo y su entorno, como se ilustra en la tabla 1.

Tabla 1. Paradigmas de enfermería (fuente: elaboración propia).

\begin{tabular}{|c|c|c|c|}
\hline \multicolumn{4}{|c|}{ Paradigmas } \\
\hline & Reacción & Interacción recíproca & Acción simultánea \\
\hline Fawcett (14) & $\begin{array}{l}\text { Divide al ser humano y lo } \\
\text { visualiza como la suma de } \\
\text { sus partes biológicas, } \\
\text { psicológicas, social y } \\
\text { espiritual. }\end{array}$ & $\begin{array}{l}\text { El ser humano es holístico, } \\
\text { interactuante con el } \\
\text { ambiente, organizado y no } \\
\text { reducible. }\end{array}$ & $\begin{array}{l}\text { El ser humano es un } \\
\text { campo holístico } \\
\text { autoorganizado, más que } \\
\text { la suma de sus partes en } \\
\text { constante intercambio con } \\
\text { el ambiente. }\end{array}$ \\
\hline \multirow[b]{2}{*}{ Smith y Liehr (6) } & Particular determinista & Interactivo integrativo & Unitario transformativo \\
\hline & $\begin{array}{l}\text { Visualiza a los fenómenos } \\
\text { como entidades } \\
\text { separables, reducibles, } \\
\text { medibles y como } \\
\text { consecuencia de un } \\
\text { antecedente causal. }\end{array}$ & $\begin{array}{l}\text { Visualiza a la realidad de } \\
\text { manera multifacética y } \\
\text { contextual, reconoce tanto } \\
\text { fenómenos de interés } \\
\text { objetivos como subjetivos y } \\
\text { las relaciones entre sus } \\
\text { partes. }\end{array}$ & $\begin{array}{l}\text { Visualiza al ser humano } \\
\text { como un ser uniforme y } \\
\text { evolucionado, que se } \\
\text { desarrolla a través de la } \\
\text { unidad e interacción con el } \\
\text { medio ambiente como un } \\
\text { todo más grande. }\end{array}$ \\
\hline \multirow[b]{2}{*}{ Kérouac (15) } & Categorización & Integración & Transformación \\
\hline & $\begin{array}{l}\text { Los fenómenos son } \\
\text { divisibles en categorías, } \\
\text { clases o grupos. } \\
\text { Fragmenta a la persona en } \\
\text { busca de un factor causal. }\end{array}$ & $\begin{array}{l}\text { Reconoce los elementos y } \\
\text { las manifestaciones de un } \\
\text { fenómeno. Visualiza la } \\
\text { globalidad de la persona. }\end{array}$ & $\begin{array}{l}\text { Los fenómenos son únicos, } \\
\text { algunos con similitudes } \\
\text { pero diferentes e } \\
\text { irrepetibles. Visualiza a la } \\
\text { persona como una unidad } \\
\text { global en interacción con el } \\
\text { mundo que la rodea. }\end{array}$ \\
\hline
\end{tabular}

Modelos conceptuales. Este apartado es concebido por Fawcett como el conjunto de imágenes y conceptos parcialmente abstractos y generales que conducen a los fenómenos de interés para enfermería (14). Con respecto a ello, Kérouac finaliza su propuesta teórica en este segmento, en donde logra engranar los modelos conceptuales como reflejo de la riqueza del saber enfermero y 
guías para la práctica, formación, investigación y gestión del cuidado en seis escuelas del conocimiento, en correspondencia a su época y visión metaparadigmática en: escuela de las necesidades, escuela de la interacción, escuela de los efectos deseables y escuela de la promoción de la salud, acopiadas en el paradigma de integración; escuela del ser humano unitario y escuela del caring, agrupadas en el paradigma de transformación (15).

Es entonces, que las escuelas del pensamiento han permitido vislumbrar los avances de la disciplina a través del tiempo y en medida de cómo ofrece sus servicios a la sociedad, a fin de clarificar y delimitar las áreas de responsabilidad práctica y como guías de entrelazamiento entre los elementos meta y paradigmáticos del quehacer disciplinario.

Teorías. Fawcett define este segmento como el conjunto de uno o más conceptos relativamente concretos y específicos, derivados de un modelo conceptual, clasificados por su nivel de abstracción en grandes teorías y teorías de mediano rango (14). A su vez concordante con lo propuesto por Smith y Liehr, quienes además adicionan como elemento de mayor abstracción las teorías prácticas y tipifican estos tres apartados por su nivel de profundidad en categorías acordes a los tres paradigmas de enfer- mería (particular-determinista, interactivo-integrativo y unitario-transformativo), con intención de que estas posturas filosóficas permitan dar significado y sentido a la amplia gama de propuestas teóricas disponibles (6). De manera que este apartado describe, explica y/o predice los problemas del quehacer disciplinario en función de empatar los fenómenos teóricos con la cotidianidad práctica mediante perspectivas específicas y en situaciones demandantes de intervención, en términos de cuidado enfermero.

Indicador empírico. Es el quinto y último apartado de la estructura jerárquica, en el que ambas propuestas teóricas (Fawcett, y Smith y Liehr) lo conciben como el vínculo entre la teoría y la práctica, que permite analizar matemáticamente, observar y medir la realidad a través de instrumentos de investigación y clínicos para un fenómeno específico (14) (6). Dicho esto, es a través de este elemento que el quehacer enfermero logra consolidar su actuación ante los ojos del mundo, al pasar de la distante y a veces frecuente invisibilidad teórica, al operacionalizar la praxis mediante pruebas empíricas que polarizan y visibilizan a la disciplina a través de indicadores de cuidado de enfermería.

Es entonces, que a partir de estas tres propuestas estructurales, no como contraste de posturas antagónicas ni con 
el propósito de legitimar una ante la otra, que se logran atisbar los elementos que sistematizan los tópicos teóricos de la disciplina, al conseguir ensamblar los componentes medulares que sostienen y guían el actuar enfermero a fin de visibilizar el conjunto de saberes científicos que facultan a sus integrantes para seguir en el desarrollo del conocimiento y posicionamiento ante las diversas corrientes cognitivas que disponen para su aprendizaje, crecimiento y para su desempeño como profesionistas.

No obstante, Smith y Liehr mencionan que diversos autores se mantienen como figuras de contrapeso ante la diferenciación de saberes y al establecimiento de los límites disciplinarios de enfermería, apostando al enriquecimiento científico del trabajo interdisciplinario (6), sin embargo, previo a la colaboración del gremio en equipos multidisciplinarios, es fundamental que se parta de visualizar y clarificar el cuerpo de conocimientos que avala su ejercicio, con la finalidad de orientar y asignar un lugar específico ante los retos de su desempeño, el de cuidar la salud de la humanidad y el mundo.

Así pues, como resultado del anterior contraste de estructuras teóricas, se presenta un diagrama en escalera en favor de armonizar y facilitar la integración de los elementos anteriormente descritos, que parte del escalón de mayor abstracción en un movimiento descendente hasta lograr llegar al escalón de mayor concreción (figura 1). 


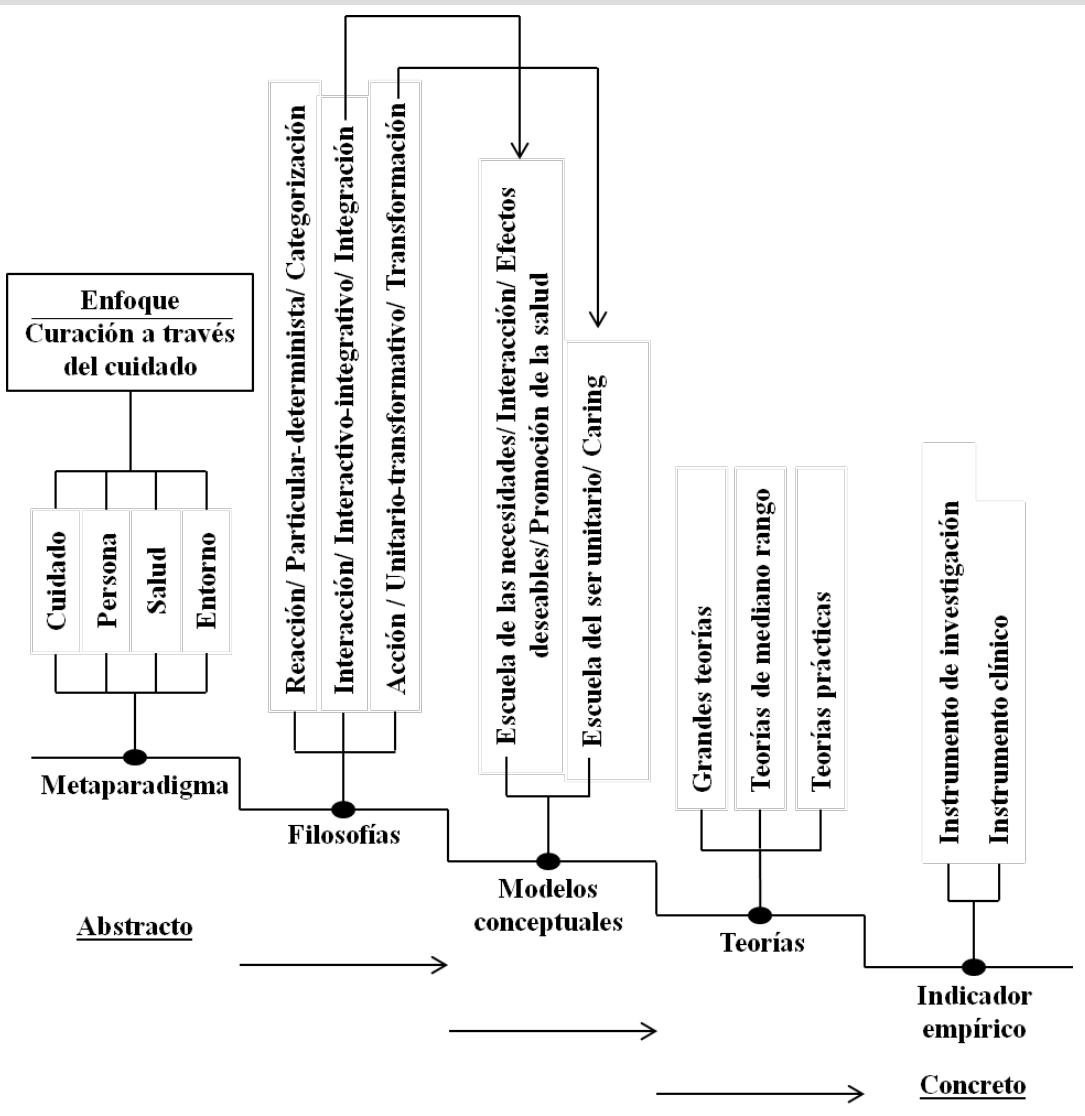

Figura 1. Escalera del conocimiento teórico de enfermería (fuente: elaboración propia).

\section{El arte de enfermería}

La conceptualización de la disciplina como arte, enfatiza el aspecto más práctico de la profesión, el acto de brindar cuidados en alusión a poner en uso el cúmulo de conocimientos que sustentan y fundamentan el actuar profesional de enfermería. Así que, si consideramos que el arte es un conocimiento práctico, manifiesto en la relación interpersonal y en la utilización de técnicas y procedimientos, el conocimiento de cómo ejecutar determinadas acciones es el medio de expresión de enfermería que permite potenciar al individuo y llegar a la realización a través del quehacer práctico (8).
En este sentido, Boykin y Schoenhofer concuerdan en que las situaciones de enfermería, consideradas como las experiencias de vida compartida entre la enfermera y los usuarios, son el depósito del conocimiento enfermero puesto que permiten reflejar el actuar y la terapéutica del cuidado (16)(17), dado que el cuidado y su significado residen en el quehacer práctico, vía de manifestación del conocimiento; por lo que su planteamiento en cuestión radica en ello y en que la práctica es el reflejo del marco epistemológico de enfermería (18).

Desde 1978 se han creado diversas propuestas que representan las formas 
de conocer de la disciplina, mismas que han permitido cristalizar y clasificar el conocimiento existente (4), no para aumentarlo, sino para centrar su interés en el significado de conocer y sobre qué tipos de conocimiento proporcionan mayor ayuda para la práctica, esencia profesional de las disciplinas (18). Adscribiéndose a lo anterior, Kim reconoció cinco dimensiones representativas de la práctica de enfermería: la dimensión científica, dimensión técnica, dimensión ética, dimensión estética y la dimensión existencial (19). Asimismo, Carper identificó cuatro formas de conocer en la praxis disciplinaria, designadas patrones del conocimiento empírico, estético, personal y ético (20); Benner sumó el patrón de experiencia (21); Young el de intuición (22); Munhall el de desconocimiento (23); White adicionó el socio-político (24) y por último, Chinn y Krammer incluyeron el patrón emancipatorio (25).

Con base en lo anterior, se tiene como premisa que el conocimiento práctico es en gran medida inarticulado, está implícito y se manifiesta en la acción (9), de manera que el quehacer enfermero como práctica de servicio, cuya razón de ser radica en mejorar las experiencias de salud humana, debe ser guiado por un sistema de conocimientos de enfermería que incluya varios tipos de conocimiento, dado que la práctica es deliberativa, está diseñada y destinada a abordar los objetivos y las necesidades particulares de las personas, familias, grupos y/o comunidades (19). De esta forma, para efectos de este apartado del artículo se retomaron las cinco dimensiones de la práctica de enfermería propuestas por Kim, como estructura discursiva del quehacer práctico, manifestadas a través de los diversos patrones del conocimiento, en aras de exponer y tangibilizar el cuidado, y como elementos imprescindibles para el progreso de la disciplina (26).

Dimensión científica. Representa la selección y aplicación de teorías científicas basadas en la racionalidad, la lógica y el poder explicativo que permite responder a las preguntas clínicas de una situación específica, satisfaciendo las necesidades cognitivas de la práctica (19).

Partiendo desde la óptica del delineamiento y organización de la multiplicidad de saberes disciplinarios de enfermería, es fundamental considerar a la dimensión científica como el conjunto de conocimientos que integran las pautas de actuación profesional que robustecen, guían y fundamentan el actuar enfermero, a partir de un desempeño profesional a priori, en donde a través del patrón empírico, la enfermera emplea leyes generales y teorías con la intención de describir, explicar y predecir fenómenos 
de interés específicos para la disciplina (20).

Dimensión técnica. Representa la manera en que las técnicas se aplican a situaciones específicas. Se traduce en términos de optimizar, coordinar, contextualizar y flexibilizar el actuar procedimental en busca de la eficacia y eficiencia para resolver los problemas de las personas y atender la particularidad de sus necesidades (19).

De forma que en esta dimensión, se evidencia la articulación del patrón empírico como punto de aprobación al conocimiento, con el patrón personal, considerado el patrón más importante para lograr comprender las experiencias de salud y el significado de bienestar, en donde se atisba la singularidad de la persona al centrarse en la subjetividad del momento, al implicar conocerse y conocer a los demás para promover la integridad del encuentro y fundar relaciones que enfrenten al ser humano como un todo y contribuyan a su salud individual (20).

Dimensión ética. Rige la racionalidad moral a partir de la cual se eligen y efectúan las acciones de cuidado en la práctica de enfermería, partiendo de la comprensión holística, la veracidad y la compasión como base normativa que aborde las obligaciones de la enfermera ante el servicio, la vulnerabilidad de las personas y la población en el mundo (19).

Es entonces, a través del patrón éti$c o$, que se comprende lo que es bueno, lo que se desea, lo que es correcto y lo que se debe hacer para preservar la vida, mitigar el sufrimiento y promover la salud (20), fundamentado en los valores personales, institucionales y humanísticos de la profesión (27). De igual manera, es a través del patrón socio-político que se faculta la creación de normas y políticas institucionales donde se funge como agente de cambio político y social con un gran compromiso por defender la salud (28). Al igual que el patrón emancipatorio, en donde se cristaliza la capacidad de reflexión en términos sociales, políticos y culturales, para lograr identificar problemas de injusticia e inequidad dentro de las instituciones y para crear condiciones justas y equitativas para todos (25).

Dimensión estética. Integra la racionalidad estética de las características basadas en la expresión de la práctica, mediante la imaginación, la armonía, el ajuste y diseño de acciones de enfermería individualistas, creativas y armoniosas dentro de una situación clínica específica (19).

Parece imprescindible que al hablar de términos meramente artísticos, subyacente en ello esté incluir locuciones concernientes a los modos de visualizar 
y entender el arte por sí mismo, en circunstancias reales. Es por ello que el patrón estético, el cual implica un profundo reconocimiento del significado de una circunstancia y como la enfermera implementa recursos creativos para modificarla, que se emplean acciones, conductas e interacciones personales en el proceso del cuidado (29), para satisfacer las necesidades de la persona, restablecer y ampliar sus capacidades, a través del actuar artístico de enfermería (20).

Dimensión existencial. Representa la racionalidad práctica de las características basadas en la experiencia, partiendo de la contextualización, la individualidad y la perspicacia de la enfermera, que permite al profesional abordar y solucionar problemas ante la singularidad y variabilidad de los asuntos humanos a través del heurismo (19).

Los diversos entornos de desempeño práctico y los múltiples retos a los que se enfrenta la enfermera en el quehacer diario, le han permitido idear y facultar las soluciones más viables durante el desempeño de sus funciones profesionales, partiendo de lo a priori a través del refinamiento de lo a posteriori. Es entonces que en esta última dimensión del cuidado se manifiestan y entretejen los tres patrones del conocimiento restantes, el patrón de experiencia en el que se transforman las expectativas teóricas preconcebidas a través de encuentros con situaciones prácticas reales y no solo como resultado del paso del tiempo (21), puesto que materializa el "saber cómo" resultado de la exposición repetida a situaciones que conducen al refinamiento de ideas y pensamientos conducidos por la intuición (30).

En consecuencia, el patrón de intuición, en el que la enfermera sabe sin saber cómo, le permite abrirse a sí misma para sentir y comprender las situaciones y respuestas de las personas (10). No sin antes partir del patrón de desconocimiento, en donde se interactúa con total ignorancia sobre la vida del otro (23), se promueve la apertura, la empatía y comprensión en esencia de la situación de la persona (30), al exhortar a reflexionar sobre el conocimiento limitado de los demás, sin comprender el grado, la extensión y la naturaleza de lo que se sabe (10).

La naturaleza práctica de enfermería y su conocimiento, fruto de la racionalidad práctico-reflexiva y resultado del actuar clínico en términos del cuidado integral profesionalizado, se desarrolla en un proceso espiral hermenéutico, es decir, de reflexión en la acción (31), en donde la estructura jerárquica permite a la enfermera discernir de la vasta gama de conocimientos multidisciplinares y disciplinares que la guían, definir y contextuali- 
zar sus actividades a través de posturas teóricas que fundamentan su ejercicio profesional de carácter autónomo e independiente. Asimismo, la organización diversificada del conocimiento en dimensiones y patrones que coexisten e interactúan entre sí, permite sistematizar la legitimidad epistémica de la disciplina en una relación conjunta de saberes que sintetizan y justifican las funciones prácticas de la profesión

(31)(32) (figura 2).

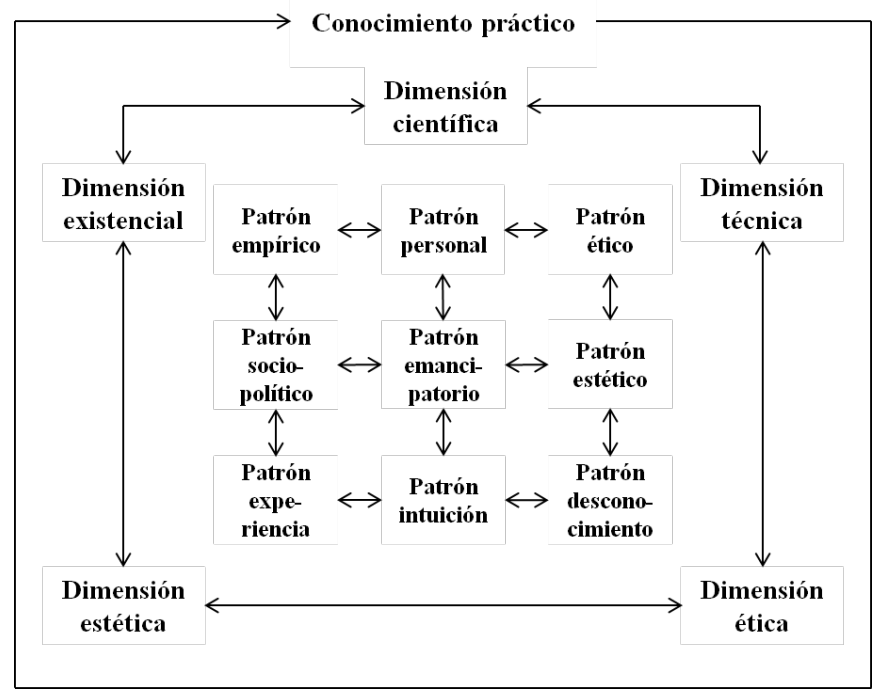

Figura 2. Diagrama del conocimiento práctico de enfermería (fuente: elaboración propia).

\section{CONSIDERACIONES FINALES}

Resulta inevitable aceptar que los enfoques actuales del conocimiento enfermero continuarán cambiando con el tiempo y en la medida que se modifiquen los valores y recursos de la sociedad (25). Asimismo, se hace evidente la necesidad de promover conexiones de intersubjetividad centradas en el quehacer prácticoreflexivo, de manera que la responsabilidad moral y los valores que guían el actuar profesional fomenten el bienestar de la población por medio de indicadores que midan, contribuyan y aseguren la calidad y calidez del cuidado humanizado.

En este sentido, algunos autores sugieren pasar de la enfermería de práctica basada en evidencias (EPBE), a la enfermería de práctica basada en el conocimiento y la enfermería de práctica basada en valores (EPBC-EPBV) ${ }^{(33)}$, dado que la convergencia de estas posturas epistemológicas en contraste al antagonismo de la EPBE, permite a la disciplina alejarse de un tipo de conocimiento, el técnico y centrado en la evidencia, y pasar a una visión más amplia e integrado- 
ra acompañada de valores éticos, sensi-

bilidad, intuición, experiencia y reflexión; características que permitirán contextualizar el saber de enfermería, con relevancia hacia el valor simbólico del acto del cuidado, en la ausencia o presencia de palabras, gestos, actitudes y emociones con un enfoque guiado por los vínculos de intersubjetividad con los individuos.

Es entonces, que ante el posible riesgo disciplinario de mantener un enfoque centrado en el tecnicismo, la racionalidad de la EPBE requiere un redireccionamiento a la visión reflexiva de la EPBCEPBV en la que converjan y contribuyan la estructura jerárquica del conocimiento, las dimensiones de la práctica de enfermería y cada uno de los patrones del conocimiento (33), ya que en conjunto, estos elementos permiten desarrollar la autonomía profesional y ampliar el campo de acción de enfermería, en el que la disciplina no se limite al quehacer técnico (28), sino todo lo contrario, se amplíe hacia un proceso de interacción epistémica, ontológica y axiológica, dirigida hacia el cuidado científico de los otros en sus procesos de salud, enfermedad y muerte (29). 


\section{BiBLIOGRAFÍA}

1. Borré-Ortiz, Y. M., Lenis-Victoria, C., Suárez-Villa, M., y Tafur-Castillo, J. (2015). El conocimiento disciplinar en el currículo de enfermería: una necesidad vital para transformar la práctica. Revista Ciencias de la Salud, 13(3), 481-491. Consulta 8 agosto, 2018, de la World Wide Web: https://bit.ly/2yTyrOR

2. Ayala, R. A. (2013). Enfermería como profesión: en busca de nuevas preguntas. Ciencia y Enfermería, 19(2), 11-21. Consulta 8 agosto, 2018, de la World Wide Web: https://bit.ly/2qj2ZY3

3. Landeros-Olvera, E., Amador-Gómez, M. R., y Pérez-Marín, M. (2018). Enfermería: de la ocupación a la profesión, de la profesión a la ciencia. Salud y Administración, 5(14), 35-42. Consulta 9 agosto, 2018, de la World Wide Web: https://bit.ly/2CTwx69

4. Siles, J. (2016). La utilidad práctica de la epistemología. Index de Enfermería, 25(1-2), 86-92. Consulta 9 agosto, 2018, de la World Wide Web: https:// bit.ly/2sARhMv

5. Kim, H. S. (2010). The nature of theoretical thinking in nursing. Springer Publishing Company, New York.

6. Smith, M. J., y Liehr, P. R. (2014). Middle range theory nursing. Springer Publishing Company, New York.

7. Trejo, F. (2013). Enfermería: del arte a la ciencia o de la ciencia al arte. Enf Neurol (Mex), 12(1), 95-97. Consulta 11 marzo, 2019, de la World Wide Web: https://bit.ly/2yVRQjD

8. Salamanca, A. B. (2017). La enfermería como arte y sus implicaciones en nuestro desarrollo colectivo. Nure Inv., 14(90), 1-2. Consulta 11 marzo, 2019, de la World Wide Web: https://bit.ly/2FjRxCF

9. Sarvimaki, A. (1994). Science and tradition in the nursing discipline. Scandinavian Journal of Caring Sciences, 8, 137-142. Consulta 10 agosto, 2018, de la World Wide Web: https://bit.ly/2F1mjTY

10. Meleis, A. I. (2012). Theoretical nursing development \& progress. Lippincott Williams \& Wilkins, Philadelphia.

11. Bueno, L. S. (2011). Aspectos ontológicos y epistemológicos de las visiones de enfermería inmersas en el quehacer profesional. Ciencia y enfermería, 17(1), 37-43. Consulta 10 agosto, 2018, de la World Wide Web: https://bit.ly/2HrtPoe

12. McEwen, M., y Wills, E. M. (2014). Theoretical basis for nursing. Lippincott Williams \& Wilkins, Philadelphia.

13. Durán de Villalobos, M. M. (2002). Marco epistemológico de la enfermería. Revista Aquichan, 2(2), 718. Consulta 10 agosto, 2018, de la World Wide Web: https://bit.ly/1cli1ND

14. Fawcett, J., y Desanto-Madeya, S. (2013). Contemporary nursing knowledge: Analysis and evalua- tion of nursing models and theories. F.A. Davis Company, Philadelphia.

15. Kérouac, S., Pepin, J., Ducharme, F., Duquette, A., y Major, F. (2007). El pensamiento enfermero. Elsevier Masson, Barcelona.

16. Boykin, A., y Schoenhofer, A. O. (2001). Nursing as caring: a model for transforming practice. Jones and Bartlett Publishers, United States of America.

17. Briñez, K. J. (2014). Narrativa de enfermería: visión y patrones del conocimiento en una entrevista en el hogar. Revista Colombiana de Enfermería, 9(9), 142-148. Consulta 15 agosto, 2018, de la World Wide Web: https://bit.ly/2OdjKgB

18. Durán de Villalobos, M. M. (2005). La ciencia, la ética y el arte de enfermería a partir del conocimiento personal. Revista Aquichan, 5(1), 86-95. Consulta 13 agosto, 2018, de la World Wide Web: https://bit.ly/ 2rESWO3

19. Kim, H. S. (2012). The role of theory in clinical nursing practice. Klinisk Sygepleje, 26(2), 16-29. Consulta 02 marzo, 2019, de la World Wide Web: https://bit.ly/2FoC7OH

20. Carper, B. A. (1978). Fundamental patterns of knowing in nursing. Advances in nursing science, 1(1), 13-24. Consulta 14 agosto, 2018, de la World Wide Web: https://bit.ly/2FEFqmm

21. Benner, P., y Wrubel, J. (1982). Skilled clinical knowledge: the value of perceptual awareness. Nurse Educator, 7(3), 11-17. Consulta 14 agosto, 2018, de la World Wide Web: https://bit.ly/2EUxVYO

22. Young, C. E. (1987). Intuition and nursing process. Holistic Nursing Practice, 1(3), 52-62. Consulta 14 agosto, 2018, de la World Wide Web: https://bit.ly/ 2zdVBRL

23. Munhall, P. L. (1993). 'Unknowing': Toward another pattern of knowing in nursing. Nursing Outlook, 41(3), 125-128.

24. White, J. (1995). Patterns of knowing: Review, critique, and update. Advances in Nursing Science, 17(4), 73-86. Consulta 14 agosto, 2018, de la World Wide Web: https://bit.ly/2RnEwfF

25. Chinn, P. L., y Krammer, M. K. (2015). Knowledge development in nursing. Elsevier, St. Louis.

26. Escobar-Castellanos, B., y Sanhueza-Alvarado, O. (2018). Patrones del conocimiento de Carper y expresión en el cuidado de enfermería: estudio de revisión. Enfermería: Cuidados Humanizados, 7(1), 57-72. Consulta 13 agosto, 2018, de la World Wide Web: https://bit.ly/2OfGc9l

27. Hernández, D. V. (2013). Una experiencia fundamental para mejorar el arte y la ciencia de cuidar. Revista Ciencia y Cuidado, 10(1), 59-66. Consulta 15 agosto, 2018, de la World Wide Web: https://bit.ly/ 2Q9hvgc

28. Osorio, J. H. (2016). Patrón de conocimiento socio-político en enfermería: reflexiones conceptua- 
les. Rev Cuid, 7(2), 1352-1357. Consulta 16 agosto, 2018, de la World Wide Web: https://bit.ly/2Q6cDbB

29. Contreras, V. (2013). Evidencia del arte en enfermería. Enfermería Global, (30), 326-331. Consulta 15 agosto, 2018, de la World Wide Web: https://bit.ly/ 2Oez90p

30. Zander, P. E. (2007). Ways of knowing in nursing: the historical evolution of a concept. The Journal of Theory Construction \& Testing, 11(1), 7-11. Consulta 16 agosto, 2018, de la World Wide Web: https://bit.ly/ 2ERY22h

31. Pina, P. J. (2016). Enfermagem, uma ecología de saberes. Cultura de los cuidados, 20(45), 137-146. Consulta 16 agosto, 2018, de la World Wide Web: https://bit.ly/2EQ7KIK

32. Pina, P. J. (2016). El conocimiento de enfermería y la naturaleza de sus saberes. Escola Anna Nery, 20(3). Consulta 17 agosto, 2018, de la World Wide Web: https://bit.ly/2qlOHG2

33. Pina, P. J. (2018). Da prática baseada em evidências à prática baseada em valores. Revista Baiana de Enfermagem, 1-3. Consulta 17 agosto, 2018, de la World Wide Web: https://bit.ly/2yKYEBk

26. Kirkpatrick A, Cantrell M, Smeltzer S. Relationships among nursing student palliative care knowledge, experience, self-awareness, and performance: An end-of-life simulation study. Nurse Educ Today. 2019 Feb; 73: p. 23-30.

27.Espinoza Venegas M, Luengo Machuca L, Sanhueza Alvarado $O$. Attitudes held by Chilean nursing professionals on care of the dying. A multivariate analysis. Aquichan. 2016; 16(4): p. 430-446.

28.Morita T, Murata $\mathrm{H}$, Hirai K, Tamura K, Kataoka J, Ohnishi $\mathrm{H}$, et al. Meaninglessness in terminally ill cancer patients: a validation study and nurse education intervention trial. J Pain Symptom Manage. 2007; 34(2): p. 160-70.

29.Watson Alvaro A. Critical care nurse's attitudes towards the care of the dying: an educational intervention. Degree of Master of Science in Nursing. Western Carolina University, Nursing Department; 2009.

30.Liping W, Jing C, Yanli D, Zhenzhen W, Zhifang L, Zhaoyang D. Factors Influencing Chinese nursing Students' Attitudes Toward the Care of Dying Patients. Journal of Hospice \& Palliative Nursing. 2017 Aug; 19 (4): p. 343-350.

31.Garrino L, Contratto C, Massariallo P, Dimonte V. Caring for dying patient and their families: the lived experiences of nursing students in Italy. Journal of palliative Care. 2017 Nov; 32(3-4): p. 127-133.

32.Draper P, Holloway M, Adamson S. A qualitative study of recently bereaved people's beliefs about death: implications for bereavement care. J Clin Nurs. 2014 May; 23(9-10): p. 1300-8.

33. Hagelin C, Melin-Johansson C, Henoch I, Bergh I, Ek K, Hammarlund $\mathrm{K}$, et al. Factors influencing attitu- de toward care of dying patients in first-year nursing students. Int J Palliat Nurs. 2016 Jan; 22(1): p. 28-36.

34. Paque $K$, Elseviers $M$, Vander Stichele R, Pardon K, Hjermstad MJ, Kaasa S, Dilles T, De Laat M, Van Belle S, Christiaens T, Deliens L. Changes in medication use in a cohort of patients with advanced cancer: The international multicentre prospective European Palliative Care Cancer Symptom study. Palliat Med. 2018 Apr; 32(4): p. 775-785.

35.Álvarez Mazariegos, JA; Calvete Waldomar, S; Fernández-Marcote Sánchez-Mayoral, RM; Guardia Serecigni, J. Guía de consenso para el buen uso de analgésicos opioides. Gestión de riesgos y beneficios. Valencia: SECPAL-SEMFyC-FAECAP; 2017.

36. White D, Meeker M. Guiding the process of dying: the personal impact on nurses. J Hosp Palliat Nurs (Publish Ahead of Print). 2019 Oct; 21(5): p. 390-396. 